\title{
Neue Option für Karzinomtherapie
}

\section{Hier steht eine Anzeige.}

\author{
Wenn Radiojod bei differenzierten \\ Schilddrüsenkarzinomen nicht \\ hilft, treten Tyrosinkinasehemmer \\ auf den Plan.
}

In Deutschland erhalten jährlich etwa 7200 Menschen die Diagnose Schilddrüsenkrebs. Mit einem Anteil von etwa 90\% sind die differenzierten Karzinome (60\% papillär, 30\% follikulär) am häufigsten. Selten sind dagegen mit etwa 5\% die medullären Karzinome, die aus Calcitoninproduzierenden Zellen entstehen.

\section{Kinasehemmer als alternative Option}

Wenn Op. und Radiojodtherapie nicht zum Erfolg führen, gibt es inzwischen die Option, die Patienten mit einem Kinasehemmer zu behandeln.

Bei Patienten mit einem progressiven Schilddrüsenkarzinom, das mit Radiojod nicht behandelt werden könne, seien die Behandlungsoptionen bislang sehr begrenzt gewesen, so Professor Dagmar Führer, Direktorin der Klinik für Endokrinologie und Stoffwechselerkrankungen am Uniklinikum Essen beim Symposium der Endokrinologen in Lübeck.
Klassische, traditionelle Chemotherapien, die bei anderen Krebsformen gute Ergebnisse erzielen, seien bei Schilddrüsenkrebs nahezu wirkungslos.

\section{Als erstes zugelassen: Sorafenib}

Das erste zugelassene Präparat aus der Klasse der Tyrosinkinasehemmer ist der Multikinasehemmer Sorafenib (Nexavar"), der bei progressivem, lokal fortgeschrittenem oder metastasiertem, differenziertem Schilddrüsen-Ca. indiziert ist, wenn die Patienten therapierefraktär gegenüber radioaktivem Jod sind. Ebenfalls in dieser Indikation zugelassen ist Lenvatinib $\left(\right.$ Lenvima $\left.{ }^{\circ}\right)$, das erst vor wenigen Wochen die EU-Zulassung erhalten hat.

Schließlich steht bereits seit 2012 Vandetanib (Caprelsa') für die Therapie zur Verfügung, allerdings für eine etwas andere Indikation. Es ist indiziert für die Behandlung von Patienten mit einem aggressiven und symptomatischen medullären Schilddrüsenkarzinom, und zwar bei nicht resektabler, lokal fortgeschrittener oder metastasierter Erkrankung. (ple)

58. Symposiums der Deutschen Gesellschaft für Endokrinologie, Lübeck; www.aerztezeitung.de

\section{In Assoziation zur Schilddrüse}

\section{Senkt Hormonbehandlung das Darmkrebsrisiko?}

Patienten mit einer Schilddrüsenfunktionsstörung, die eine Hormontherapie erhalten, haben möglicherweise ein verringertes Risiko, an Darmkrebs zu erkranken.

Diese bereits in einer früheren Studie festgestellte Assoziation haben britische Gastroenterologen in einer bevölkerungsgestützten Studie mit mehr als
100.000 Teilnehmern bestätigt. Bei Patienten, die über einen Zeitraum von mehr als zehn Jahren behandelt worden waren, war die Wahrscheinlichkeit, ein kolorektales Karzinom zu entwickeln, um 32\% verringert (adjustierte Odds Ratio: 0,68 (95\%-Konfidenzintervall $\mathrm{zwi}$ schen 0,55 und 0,$83 ; \mathrm{p}<0,01$ ).

(ple) 